\title{
Minimally Invasive Glaucoma Surgery - to Remove or Preserve the Trabecular Meshwork: That is the Question?
}

\author{
Tanuj Dada ${ }^{1}$, Karthikeyan Mahalingam ${ }^{2}$, Shibal Bhartiya ${ }^{3}$ \\ Journal of Current Glaucoma Practice (2021): 10.5005/jp-journals-10078-1299
}

The aqueous drainage structures, trabecular meshwork (TM) and Schlemm's canal (SC), are derived from the periocular mesenchyme and are the last structures to differentiate during the anterior segment development. ${ }^{1-3}$ Trabecular meshwork is a tiny porous triangular structure, which acts as a filter between the anterior chamber and SC. ${ }^{4}$

The aqueous drains through the efferent channels of the circular canal of Schlemm, which is about $25 \mathrm{~cm}$ long, into the episcleral venous plexus at the level of the Tenon's capsule and the conjunctiva. It then drains into the superior ophthalmic vein and enters the cavernous sinus above the annulus of Zinn, and then into the right atrium via the superior vena cava and the internal jugular vein. ${ }^{3}$

The intraocular pressure (IOP), therefore, is determined by a dynamic play of the following parameters: IOP is the rate of aqueous humor production divided by the facility of outflow plus episcleral venous pressure (EVP).

In glaucoma patients, the pores of the TM get blocked by debris leading to an increase in resistance to outflow of aqueous.

\section{What is the Function of Trabecular Meshwork-Schlemm's Canal Complex?}

It is important to think about "why did mother nature place this intricate multilayer TM over the SC". It could have let the aqueous directly drain into the SC without the need for a complex drain over it. Without understanding this complex function, we may be doing great harm in removing the TM as a primary strategy to lower IOP.

In principle, when a sieve/strainer over a drainage system gets blocked there are two options-either we clean the sieve or we put a new sieve. If we remove the sieve, in a matter of time the entire drainage system will get clogged as the debris will now move downstream and cause obstruction at a deeper level. However, this is exactly what we may be doing in removing the TM.

For example, can the dumping of inflammatory debris from the anterior chamber in uveitic glaucoma, without the intervention of the active molecular sieve, directly into the systemic circulation have potentially deleterious effects, like providing a nidus for inflammatory plaque formation?

\section{The TM Complex is an Active Aqueous PUMP}

The traditional concept states that the flow of aqueous from the anterior chamber into TM and SC is a passive process, but the

\begin{abstract}
1,2 Dr Rajendra Prasad Centre for Ophthalmic Sciences, All India Institute of Medical Sciences, New Delhi, India

${ }^{3}$ Department of Ophthalmology, Glaucoma Services, Fortis Memorial Hospital, Gurugram, Haryana, India

Corresponding Author: Tanuj Dada, Dr Rajendra Prasad Centre for Ophthalmic Sciences, All India Institute of Medical Sciences, New Delhi, India, Phone: +91 9873336315, e-mail: tanujdada@gmail.com

How to cite this article: Dada T, Mahalingam K, Bhartiya S. Minimally Invasive Glaucoma Surgery-to Remove or Preserve the Trabecular Meshwork: That is the Question? J Curr Glaucoma Pract 2021;15(2): 47-51.

Source of support: Nil

Conflict of interest: None
\end{abstract}

current evidence suggests that it is an active phenomenon driven by a mechanical pump. ${ }^{5,6}$ The systole of the cardiac cycle, blinking of an eyelid, and movement of the eye cause a transient increase in IOP powering the aqueous pump.

\section{Tissue Relationship}

The cells lining trabecular lamellae and the SC endothelial cells extend cytoplasmic processes, attaching with the juxtacanalicular cell processes, which acts as a cellular linking mechanism.

\section{Inlet and Outlet Valves of Schlemm's Canal}

The aqueous outflow enters SC through the inlet valves. ${ }^{6}$ The inlet valves are connected to hinged flaps of outlet valves. Experimental observations revealed that the gentle suction on TM pulls it, causing elongation of the valves. The elongation of the inlet valve opens the outlet valve. There is immediate recoil of TM and these valves when the suction is released. In vivo, microscopic deformations occur to these structures during transient spikes of IOP. Destruction of the inlet valve and its septal connection with the outlet valve can therefore lead to the closure of the outlet valve and further increase outflow resistance.

\section{IOP Homeostasis}

While the EVP remains relatively constant throughout the day, it increases in the supine position, with a corresponding, often linear increase in IOP. It is interesting to note that the EVP may be higher in patients with glaucoma (other than those associated with classical elevated EVP also) when compared with normal subjects.

During systole, the aqueous is pushed into collector channel ostia and aqueous veins due to the outward movement of the SC endothelial cells into the SC. As IOP increases during systole,

(c) Jaypee Brothers Medical Publishers. 2021 Open Access This article is distributed under the terms of the Creative Commons Attribution 4.0 International License (https://creativecommons.org/licenses/by-nc/4.0/), which permits unrestricted use, distribution, and non-commercial reproduction in any medium, provided you give appropriate credit to the original author(s) and the source, provide a link to the Creative Commons license, and indicate if changes were made. The Creative Commons Public Domain Dedication waiver (http://creativecommons.org/publicdomain/zero/1.0/) applies to the data made available in this article, unless otherwise stated. 
aqueous is forced into this one-way valve spanning the SC. During diastole, the IOP reduces and elastic recoil of the trabecular lamellae and the SC endothelial cells causes reduction of pressure in SC, causing entry of aqueous into it from SC valves. The microstructure of TM and SC is coupled with IOP, thus acting as a pressure-sensing, and pressure regulatory, setup maintaining IOP homeostasis. ${ }^{7}$

\section{Outflow System beyond the Schlemm's Canal is a Major Cause (Up to $50 \%$ ) for Resistance to Aqueous Flow}

Experimental studies on enucleated human eyes have initially suggested that approximately $75 \%$ of resistance to aqueous flow lies proximal to the SC inner wall and $25 \%$ in the outflow system beyond the canal outer wall. This cannot be applied clinically because in enucleated eyes, resistance at level of distal outflow pathway is zero/absent. However, recent studies suggest that this resistance in the outflow channels beyond the canal may be as high as $50 \%$, increasing with the severity of glaucoma. ${ }^{1,8-12}$ At perfusion pressures of $7 \mathrm{~mm} \mathrm{Hg}$, it was found that even after removal of $360^{\circ}$ of the TM, the resistance that was eliminated was only $50 \%$.

\section{Circumferential Extent of Surgery on TM does not Correlate with a Reduction in IOP}

Rosenquist et al. studied the outflow resistance in nine pairs of enucleated human eyes (with no history of glaucoma, trauma, or any glaucoma surgery) at two different (7 and $25 \mathrm{~mm} \mathrm{Hg}$ ) perfusion pressures and different extents of trabeculotomy. ${ }^{12}$ At different perfusion pressures, 1 clock hour and 4 clock hours of trabeculotomy showed $41-60$ and $79-85 \%$ of the effect (elimination of outflow resistance) produced by 12 clock hours trabeculotomy. ${ }^{12}$ Wecker et al. retrospectively observed 93 patients which had underwent trabectome surgery and concluded that the extent of trabeculotomy did not correlate with the postoperative reduction in IOP. ${ }^{13}$ Manabe et al. examined the relationship between the extent of trabeculotomy and the postoperative reduction in IOP in 48 consecutive eyes scheduled for suture trabeculotomy and concluded that the extent of incision did not correlate with the IOP reduction. ${ }^{14}$ Hirabayashi et al. compared the surgical outcomes of sectoral excisional goniotomy using Kahook Dual Blade $\left(90^{\circ}-120^{\circ}\right)$ vs $360^{\circ}$ circumferential trabeculotomy [gonioscopy-assisted transluminal trabeculotomy (GATT)/Trab360]. ${ }^{15}$ Although IOP reduction was comparable in both groups at 6 months, a higher number of eyes in sectoral incisional goniotomy achieved the target IOP compared with $360^{\circ}$ trabeculotomy group.

Hence, the extent of trabeculotomy opening is not proportional to the amount of resistance eliminated, and opening a wider area of the SC does not translate into a higher IOP reduction. In glaucomatous eyes, compared with normal eyes, the pore density in the inner wall of the SC, through which the aqueous flows is decreased, and the thickness of juxtacanalicular connective tissue is increased. ${ }^{16,17}$ Thus, the resistance to aqueous outflow is increased leading to raised IOP. Novels drugs (like Rho-kinase inhibitors) and laser therapy (like SLT) target the TM and play an important role in remodeling and reducing TM resistance and if the TM is removed we no longer have the option of using these therapies. This is especially relevant in eyes with early glaucoma and younger patients where the TM is still functional and new therapies targeting the TM may be available in the future.

\section{IOP Lowering Efficacy, Safety, and Rebound Increase in IOP after Ab Interno Trabeculotomy Procedures}

\section{Partial Trabeculotomy}

Trabeculotomy, introduced in the 1960s like trabeculectomy, is known to reduce the IOP by increasing the flow through the SC and collector channels. ${ }^{18-20}$ In this procedure, the TM and the inner wall of SC are either incised or resected to decrease the resistance to aqueous flow. ${ }^{14}$ The 21 st-century trabeculotomy has evolved from an $a b$ externo to an $a b$ interno procedure. ${ }^{14,18-30}$ It includes GATT, kahook dual blade goniotomy, microhook ab interno trabeculotomy, and modifications like bent needle ab interno goniectomy (BANG), etc. The TM is torn in GATT and excised in the others. The minimally invasive nature of these procedures holds great promise: there are no conjunctival or scleral incisions, no filtering blebs, no wound modulation woes, and of course, the touchstone of modern glaucoma surgery, they can easily be combined with cataract surgery.

Their universal adoption, however, requires a little more critical appraisal as the IOP lowering capability is very variable and achieves a target IOP usually appropriate for early glaucoma or ocular hypertension and there is a turn back elevation in IOP as scarring develops in the operated area. ${ }^{31,32}$ Tanito analyzed the midterm results of microhook $a b$ interno trabeculotomy in 560 eyes and reported a success rate of $32.1 \%$ at 2 years with glaucoma medications (criteria: IOP $\geq 18 \mathrm{~mm} \mathrm{Hg}$ and $20 \%$ or more reduction in IOP). ${ }^{27}$ Chihara and Chihara reported a similar poor success rate at 2 years with the Kahook dual blade trabeculotomy combined with phacoemulsification. ${ }^{33}$ In fact, they also found a significant turn-back elevation in IOP after the initial IOP reduction following the procedure. Ahuja et al. studied the effect of $120^{\circ} a b$ interno trabeculotomy using trabectome in 246 patients and reported that it is an appropriate procedure only when the target IOP is $21 \mathrm{~mm} \mathrm{Hg}$ or above as the success rate is only $22 \%$ at 2 years if target IOP is $<18 \mathrm{~mm} \mathrm{Hg} .{ }^{34}$ Jea et al. compared the efficacy of $a b$ interno trabeculectomy using trabectome $\left(60^{\circ}-150^{\circ}\right)$ vs trabeculectomy (with mitomycin C) and concluded that the limited ab interno trabeculectomy had a lower success rate compared with conventional trabeculectomy. ${ }^{35}$

\section{Complete $360^{\circ}$ Trabeculotomy}

Fontana et al. compared outcomes of GATT vs trabeculectomy with mitomycin $C$ in patients older than 18 years and concluded that the IOP lowering effect of trabeculectomy was higher than GATT. ${ }^{36}$ Their use, therefore, may be limited to mild to moderate cases of glaucoma and in patients with ocular hypertension. They might not be appropriate for severe glaucoma with a target IOP $<15 \mathrm{~mm} \mathrm{Hg}$.

The most common postoperative complication, seen in $30-40 \%$ of the cases following ab-interno trabeculotomy procedures, is hyphema. ${ }^{22,27,33,37}$ Espinoza et al. reported a case of bilateral delayed onset hyphema after GATT triggered by pupillary dilation. ${ }^{32}$ Bektas et al. reported that the macrohyphema negatively influences the success rate after GATT. ${ }^{38}$ Blood 
reflux can also happen with any Valsalva maneuver like weight lifting, playing wind blowing instruments, or constipation. Due to the risk of postoperative hyphema, there might be a need to stop anticoagulants after GATT. There is a higher chance of hypotony (pressure drop below the EVP) and blood reflux from the episcleral veins causing hyphema following conventional glaucoma surgeries (both trabeculectomy and glaucoma drainage devices), in case they are performed following the failure of minimally invasive $a b$ interno trabeculotomy. No studies are evaluating the long-term outcomes of trabeculectomy in patients previously operated minimally invasive ab interno trabeculotomy. So minimally invasive $a b$ interno trabeculotomy should be cautiously used, especially in young patients who might require trabeculectomy later on.

\section{Why do Procedures on the TM Fall?}

Peripheral anterior synechiae (PAS) formation has been documented by gonio camera after microhook ab-interno trabeculotomy. ${ }^{39}$ Thus, PAS formed as a healing response can subsequently obstruct aqueous flow to SC. Amari et al. investigated the reasons for the failure of trabeculotomy using the trabeculectomy specimens (obtained in areas of the previous trabeculotomy) and observed the following changes after trabeculotomy: fusion of TM leaflets, accumulation of pigments, the membrane covering the canal, persistence of TM, damaged outflow channels. ${ }^{40}$

Aktas et al. reported a case of panscleritis following GATT in uveitis glaucoma. ${ }^{41}$ So, surgeon must be cautious and an adequate period of inactivity must be documented before performing GATT in uveitic glaucoma.

Also, the extent of incision/resection of the TM-SC complex does not correlate with the amount of IOP reduction achieved postoperatively. ${ }^{13,14}$ This could be attributed to a variable resistance in the distal outflow pathway because of the anatomical alterations, as observed by Hann et al. using three-dimensional microcomputed tomography. ${ }^{42}$ Even though Fellman et al. reported that the intraoperative episcleral venous waves correlated with the postoperative IOP reduction after trabeculotomy, ${ }^{43}$ the decidedly non-linear relationship between the extent of incision and postoperative IOP reduction means that the surgical procedure cannot be titrated to achieve target IOP.

\section{Future Perfect: Redefining "Minimally" INVASIVE}

The basic premise of MIGS is to preserve the conjunctiva for future filtration procedures, given the natural history of glaucoma. It is a chronic, progressive disease, and scarring, leading to surgical failure is inevitable, should the patient live long enough.

For now, there is no known mechanism of modulating wound healing at the level of the TM; modulation of conjunctival wound healing remains an imperfect science despite decades of research. An improved therapeutic index for the available conjunctival healing modulators remains elusive: finding a safe, specific, focal, and titratable biological molecule for the TM is still a long way away.

Strangely, in an attempt to preserve the conjunctiva, those of us who manage glaucoma, forget to preserve the TM: the site of almost all that happens in glaucoma.

We forget that future research in glaucoma management is focusing on restoring TM functionality, and that is not a utopian concept any longer. Rho-kinase inhibitors, marine macrolides, adenosine- and prostanoid-receptor agonists together with siRNA formulations have been the focus of pharmacotherapeutic research since they increase conventional aqueous outflow via modulation of the JCT or the cytoskeleton of the outflow pathway cells. ${ }^{44}$ Rho kinase (ROCK) inhibitors, and norepinephrine transporter (NET) inhibitors, have both shown promise in this regard, and many more are on the anvil. Netarsudil, the recently approved Rho-kinase inhibitor, has been found to have some anti-fibrotic activity, and can restore the stiffness/function of the TM, and thus enhances the aqueous outflow. ${ }^{45,46}$ Trabodenoson, formerly designated INO8875, an adenosine receptor (A1R) agonist is thought to enhance extracellular matrix turnover via multiple signaling pathways including matrix metalloproteinase (MMP-2), thereby increasing the outflow facility. ${ }^{47}$ Similarly, commercially available latanoprostene bunod $0.024 \%$ (Vyzulta), a prostaglandin F2a analog, is thought to lower IOP by increasing aqueous humor outflow through both, the uveoscleral pathway (mediated by latanoprost acid), and the TM pathway (mediated by donated nitric oxide). ${ }^{48}$

There has also been a remarkable interest in stem cell-based therapies for TM regeneration. Several stem cell types, such as adipose-derived stem cells, mesenchymal stem cells, induced pluripotent stem cells (iPSCs), corneal endothelium (CE), an anterior non-filtering portion of the TM called Schwalbe's ring region cells, as well as TM stem cells, can differentiate into TM cells in vivo. ${ }^{49-53}$ These cells show a TM cell-like genotype, became phagocytic, and respond to dexamethasone stimulation, like native TM cells. ${ }^{50}$ Intracamerally transplanted human trabecular meshwork stem cells (TMSCs) in mice have been shown to migrate to the site of laser trabeculoplasty via chemotaxis. ${ }^{51}$ Human iPSCs can be cultured as colonies and monolayer cells, ${ }^{52}$ and autologous or allogeneic stem cells, therefore, constitute a potential treatment to restore TM structure and function in glaucoma.

Is it not, therefore, an aberration, that all those who undergo a surgery touted to be minimally invasive keeping the future in mind, become almost ineligible for an entire class of disease-modifying drugs, and also stem cell-mediated refunctionalization of the TM, once they are available?

Does it not, therefore, stand to reason that a surgery that destroys the TM not be labeled minimally invasive, merely because it spares the conjunctiva for a future filter-because it may preclude the use of, or certainly decrease the effect of, an entire class of drugs acting on the TM?

Table 1 has some points to ponder over while considering $360^{\circ}$ trabeculotomy.

\section{The Verdict}

Sparing the TM-SC complex preserves the active aqueous pump (although it functions to a less extent in glaucomatous eyes) and allows novel drugs like Rho-kinase inhibitors to act on it. Even if the 3600 trabeculotomy (GATT) is categorized as minimally invasive, it is a very traumatic and destructive surgery with long-term risk of spontaneous hyphemas. MIGS on the TM should be restricted to a limited nasal quadrant trabeculotomy since this provides the best balance between IOP reduction and preservation of 3/4th of the TM tissue. Additionally, procedures to dilate the SC which requires only small perforation of the TM may be preferred as they enhance the function of the TM without any destruction of this vital tissue. ${ }^{54-56}$ 
Table 1: Drawbacks of $360^{\circ} \mathrm{TM}$ surgery

\section{IOP control and homeostatic mechanisms}

- Lesser IOP reduction compared with traditional glaucoma filtering surgeries

- Turn back elevation in IOP

- The outerwall of Schlemm's canal can be damaged during the procedure

- Destroys active aqueous pump

- Does not take distal outflow resistance into consideration

\section{Patient selection and preoperative considerations}

- Achieves medication-free target IOP only for early glaucoma

- Trabeculectomy required subsequently increases the risk of late spontaneous hyphema

\section{Postoperative considerations}

- Drugs enhancing the aqueous pump cannot be used postoperatively

- Selective laser trabeculoplasty cannot be done as trabecular meshwork is destroyed

- Postoperative complications like hyphema may require the patient to be in the propped up position for 2 weeks or more and adversely affect the quality of life (especially in active young individuals)

- All activities that involve the Valsalva maneuver may be contraindicated

- Use of anticoagulants may precipitate late hyphema

- Effect of uveitis - no barrier to inflammatory cells (needs to be critically evaluated)

- No long-term studies on efficacy and safety especially if subsequent trabeculectomy or GDD performed

\section{References}

1. Gould DB, Smith RS, John SWM. Anterior segment development relevant to glaucoma. Int J Dev Biol 2004;48(8-9):1015-1029. DOI: 10.1387/ijdb.041865dg.

2. Hamanaka T, Bill A, Ichinohasama R, et al. Aspects of the development of Schlemm's canal. Exp Eye Res 1992;55(3):479-488. DOI: 10.1016/0014-4835(92)90121-8.

3. Abu-Hassan DW, Acott TS, Kelley MJ. The trabecular meshwork: a basic review of form and function. J Ocul Biol 2014;2(1). DOI: 10.13188/2334-2838.1000017Available from: https://www.ncbi.nlm. nih.gov/pmc/articles/PMC4209746/

4. Buffault J, Labbé A, Hamard P, et al. The trabecular meshwork: Structure, function and clinical implications. A review of the literature. J Fr Ophtalmol 2020;43(7):e217-e230. DOI: 10.1016/j.jfo.2020.05.002.

5. Johnstone MA. The aqueous outflow system as a mechanical pump: evidence from examination of tissue and aqueous movement in human and non-human primates. J Glaucoma 2004;13(5):421-438. DOI: 10.1097/01.ijg.0000131757.63542.24.

6. Johnstone M, Xin C, Tan J, et al. Aqueous outflow regulation - 21st century concepts. Prog Retin Eye Res 2020. 100917. DOI: 10.1016/j. preteyeres.2020.100917.

7. Johnstone MA, Grant WG. Pressure-dependent changes in structures of the aqueous outflow system of human and monkey eyes. Am J Ophthalmol 1973;75(3):365-383. DOI: 10.1016/0002-9394(73)91145-8.

8. Grant WM. Further studies on facility of flow through the trabecular meshwork. AMA Arch Ophthalmol 1958;60(4 Part 1):523-533. DOI: 10.1001/archopht.1958.00940080541001.

9. Grant WM. Experimental aqueous perfusion in enucleated human eyes. Arch Ophthalmol 1963;69(6):783-801. DOI: 10.1001/archo pht.1963.00960040789022.

10. Ellingsen BA, Grant WM. Trabeculotomy and sinusotomy in enucleated human eyes. Invest Ophthalmol 1972;11(1):21-28.
11. Van Buskirk EM, Grant WM. Lens depression and aqueous outflow in enucleated primate eyes. Am J Ophthalmol 1973;76(5):632-640. DOI: 10.1016/0002-9394(73)90555-2.

12. Rosenquist $R$, Epstein $D$, Melamed $S$, et al. Outflow resistance of enucleated human eyes at two different perfusion pressures and different extents of trabeculotomy. Curr Eye Res 1989;8(12):1233-1240. DOI: $10.3109 / 02713688909013902$.

13. Wecker T, Anton A, Neuburger M, et al. Trabeculotomy opening size and IOP reduction after Trabectome ${ }^{\circledast}$ surgery. Graefes Arch Clin Exp Ophthalmol 2017;255(8):1643-1650. DOI: 10.1007/s00417-017-3683-0.

14. Manabe S-I, Sawaguchi S, Hayashi K. The effect of the extent of the incision in the Schlemm canal on the surgical outcomes of suture trabeculotomy for open-angle glaucoma. Jpn J Ophthalmol 2017;61(1):99-104. DOI: 10.1007/s10384-016-0487-4.

15. Hirabayashi MT, Lee D, King JT, et al. Comparison of surgical outcomes of $360^{\circ}$ circumferential trabeculotomy versus sectoral excisional goniotomy with the Kahook dual blade at 6 months. Clin Ophthalmol 2019;13:2017-2024. DOI: 10.2147/OPTH.S208468.

16. Johnson M, Chan D, Read AT, et al. The pore density in the inner wall endothelium of Schlemm's canal of glaucomatous eyes. Invest Ophthalmol Vis Sci 2002;43(9):2950-2955.

17. Buller C, Johnson D. Segmental variability of the trabecular meshwork in normal and glaucomatous eyes. Invest Ophthalmol Vis Sci 1994;35(11):3841-3851.

18. Smith R. A new technique for opening the canal of Schlemm. Preliminary report Br J Ophthalmol 1960;44(6):370-373. DOI: 10.1136/ bjo.44.6.370.

19. McPherson SD, McFarland D. External trabeculotomy for developmental glaucoma. Ophthalmology 1980;87(4):302-305. DOI: 10.1016/s0161-6420(80)35233-0.

20. Grehn F. The value of trabeculotomy in glaucoma surgery. Curr Opin Ophthalmol 1995;6(2):52-60. DOI: 10.1097/00055735-19950400000009.

21. Grover DS, Fellman RL. Gonioscopy-assisted transluminal trabeculotomy (GATT): thermal suture modification with a dyestained rounded tip. J Glaucoma 2016;25(6):501-504. DOI: 10.1097/ IJG.0000000000000325.

22. Grover DS, Godfrey DG, Smith O, et al. Gonioscopy-assisted transluminal trabeculotomy, ab interno trabeculotomy: technique report and preliminary results. Ophthalmology 2014;121(4):855-861. DOI: 10.1016/j.ophtha.2013.11.001.

23. Grover DS, Godfrey DG, Smith O, et al. Outcomes of gonioscopyassisted transluminal trabeculotomy (GATT) in eyes with prior incisional glaucoma surgery. J Glaucoma 2017;26(1):41-45. DOI: 10.1097/IJG.0000000000000564.

24. Richter GM, Coleman AL. Minimally invasive glaucoma surgery: current status and future prospects. Clin Ophthalmol 2016;10:189206. DOI: $10.2147 / O P T H . S 80490$.

25. Allen L, Burian HM. Trabeculotomy ab externo. A new glaucoma operation: technique and results of experimental surgery. Am J Ophthalmol 1962;53(1):19-26. DOI: 10.1016/0002-9394(62) 90388-4.

26. Quigley HA. Results, with trabeculotomy and study of reversible cupping. Ophthalmology 1982;89(3):219-226. DOI: 10.1016/s01616420(82)34803-4.

27. Tanito M. Microhook ab interno trabeculotomy, a novel minimally invasive glaucoma surgery. Clin Ophthalmol 2017;12:43-48. DOI: 10.2147/OPTH.S152406.

28. Dorairaj S, Tam MD. Kahook dual blade excisional goniotomy and goniosynechialysis combined with phacoemulsification for angleclosure glaucoma: 6-month results. J Glaucoma 2019;28(7):643-646. DOI: $10.1097 /$ IJG.0000000000001256.

29. Salinas L, Chaudhary A, Berdahl JP, et al. Goniotomy using the Kahook dual blade in severe and refractory glaucoma: 6-month outcomes. J Glaucoma 2018;27(10):849-855. DOI: 10.1097/JJ.0000000000001019.

30. Wakil SM, Birnbaum F, Vu DM, et al. Efficacy and safety of a singleuse dual blade goniotomy: 18-month results. J Cataract Refract Surg 2020;46(10):1408-1415. DOI: 10.1097/j.jcrs.0000000000000263. 
31. Conlon R, Saheb H, Ahmed IIK. Glaucoma treatment trends: a review. Can J Ophthalmol 2017;52(1):114-124. DOI: 10.1016/j.jcjo.2016. 07.013.

32. Espinoza G, Rodriguez-Una I, Pedraza-Concha A. A case of bilateral delayed-onset hyphema following pupil dilation after gonioscopyassisted transluminal trabeculotomy. J Curr Glaucoma Pract 2020;14(2):72-75. DOI: 10.5005/jp-journals-10078-1279.

33. Chihara E, Chihara T. Turn back elevation of once reduced IOP after trabeculotomy ab externo and Kahook dual blade surgeries combined with cataract surgery. OPTH 2020;14:4359-4368. DOI: 10.2147/OPTH.S287090.

34. Ahuja Y, Ma Khin Pyi S, Malihi M, et al. Clinical results of ab interno trabeculotomy using the trabectome for open-angle glaucoma: the mayo clinic series in Rochester, Minnesota. Am J Ophthalmol 2013;156(5):927-935.e2. DOI: 10.1016/j.ajo.2013.06.001.

35. Jea SY, Francis BA, Vakili G, et al. Ab interno trabeculectomy versus trabeculectomy for open-angle glaucoma. Ophthalmology 2012;119(1):36-42. DOI: 10.1016/j.ophtha.2011.06.046.

36. Fontana L, De Maria M, Caristia A, et al. Comparison of gonioscopyassisted transluminal trabeculotomy versus trabeculectomy with mitomycin C in patients with open-angle glaucoma. J Glaucoma 2021;30(1):101-108. DOI: 10.1097/IJG.0000000000001696.

37. Grover DS, Smith O, Fellman RL, et al. Gonioscopy-assisted transluminal trabeculotomy: an ab interno circumferential trabeculotomy: 24 months follow-up. J Glaucoma 2018;27(5):393-401. DOI: $10.1097 /$ IJG.0000000000000956.

38. Bektas C, Aktas Z, Ucgul AY, et al. Prognostic factors affecting the surgical success of gonioscopy-assisted transluminal trabeculotomy. Indian J Ophthalmol 2021;69(6):1425-1429. DOI: 10.4103/ijo. IJO_2035_20.

39. Matsuo $M$, Inomata $Y$, Kozuki N, et al. Characterization of peripheral anterior synechiae formation after microhook $a b$-interno trabeculotomy using a 360-degree gonio-camera. Clin Ophthalmol 2021;15:1629-1638. DOI: 10.2147/OPTH.S306834.

40. Amari Y, Hamanaka T, Futa R. Pathologic investigation failure of trabeculotomy. J Glaucoma 2015;24(4):316-322. DOI: 10.1097/ IJG.0b013e31829e1d6e.

41. Aktas Z, Bektas C, Hasanreisoglu M. Panscleritis as an unusual complication of gonioscopy-assisted transluminal trabeculotomy. J Glaucoma 2019;28(2):e21-e23. DOI: 10.1097/JJG.0000000000001100.

42. Hann CR, Bentley MD, Vercnocke A, et al. Imaging the aqueous humor outflow pathway in human eyes by three-dimensional microcomputed tomography (3D micro-CT). Exp Eye Res 2011;92(2):104111. DOI: 10.1016/j.exer.2010.12.010.

43. Fellman RL, Feuer WJ, Grover DS. Episcleral venous fluid wave correlates with trabectome outcomes: Intraoperative evaluation of the trabecular outflow pathway. Ophthalmology 2015;122(12):23852391.e1. DOI: 10.1016/j.ophtha.2015.08.038.

44. O'Callaghan J, Cassidy PS, Humphries P. Open-angle glaucoma: therapeutically targeting the extracellular matrix of the conventional outflow pathway. Expert Opin Ther Targets 2017;21(11):1037-1050. DOI: $10.1080 / 14728222.2017 .1386174$.

45. Ethier CR, Li G, Lee C, et al. Netarsudil (NT) restores trabecular meshwork (TM) function/stiffness and reduces IOP in steroidinduced ocular hypertension (OHT). Invest Ophthalmol Vis Sci 2020;10(61):2325-2325.

46. Li G, Lee C, Read AT, et al. Anti-fibrotic activity of a rho-kinase inhibitor restores outflow function and intraocular pressure homeostasis Barton M, Smith L, McDowell C, et al., ed. eLife., vol. 10 2021. p. e60831.

47. Qiu TG. Trabodenoson on trabecular meshwork rejuvenation: a comprehensive review of clinical data. Expert Opin Investig Drugs 2021;30(3):227-236. DOI: 10.1080/13543784.2021.1873276.

48. Hoy SM. Latanoprostene bunod ophthalmic solution $0.024 \%$ : a review in open-angle glaucoma and ocular hypertension. Drugs 2018;78(7):773-780. DOI: 10.1007/s40265-018-0914-6.

49. Yun $H$, Zhou Y, Wills A, et al. Stem cells in the trabecular meshwork for regulating intraocular pressure. J Ocul Pharmacol Ther 2016;32(5):253-260. DOI: 10.1089/jop.2016.0005.

50. Zhou Y, Xia X, Yang E, et al. Adipose-derived stem cells integrate into trabecular meshwork with glaucoma treatment potential. FASEB J 2020;34(5):7160-7177. DOI: 10.1096/fj.201902326R.

51. Yun $\mathrm{H}$, Wang $\mathrm{Y}$, Zhou $\mathrm{Y}$, et al. Human stem cells home to and repair laser-damaged trabecular meshwork in a mouse model. Commun Biol 2018;1(1):216. DOI: 10.1038/s42003-018-0227-z.

52. Kumar A, Cheng T, Song W, et al. Two-step induction of trabecular meshwork cells from induced pluripotent stem cells for glaucoma. Biochem Biophys Res Commun 2020;529(2):411-417. DOI: 10.1016/j. bbrc.2020.05.225.

53. Vingolo EM, Chabib A, Anselmucci F. Regeneration of trabecular meshwork in primary open angle glaucoma by stem cell therapy: a new treatment approach. Transpl Res Risk Manag 2019;11:11-16. DOI: 10.2147/TRRM.S160229.

54. Kazerounian S, Zimbelmann M, Lörtscher M, et al. Canaloplasty ab interno (AbiC) - 2-year-results of a novel minimally invasive glaucoma surgery (MIGS) technique. Klin Monbl Augenheilkd 2020. Epub ahead of print.

55. Hughes T, Traynor M. Clinical results of ab interno canaloplasty in patients with open-angle glaucoma. Clin Ophthalmol 2020;14:36413650. DOI: $10.2147 /$ OPTH.S275087.

56. Dickerson JE, Brown RH. Circumferential canal surgery: a brief history. Curr Opin Ophthalmol 2020;31(2):139-146. DOI: 10.1097/ ICU.0000000000000639. 